\title{
ACTIONS OF REDUCTIVE GROUPS ON REGULAR RINGS AND COHEN-MACAULAY RINGS
}

\author{
BY MELVIN HOCHSTER AND JOEL L. ROBERTS ${ }^{1}$
}

Communicated by Dock S. Rim, August 30, 1973

0. The main results. This note is an announcement of the results below, whose proofs will appear separately [7].

MAIN THEOREM. Let $G$ be a linearly reductive affine linear algebraic group over a field $K$ of arbitrary characteristic acting $K$-rationally on a regular Noetherian K-algebra $S$. Then the ring of invariants $R=S^{G}$ is Cohen-Macaulay.

THEOREM. If $S$ is a regular Noetherian ring of prime characteristic $p>0$, and $R$ is a pure subring of $S$ (i.e.for every $R$-module $M, M \rightarrow M \otimes_{R} S$ is injective), e.g. if $R$ is a direct summand of $S$ as $R$-modules, then $R$ is Cohen-Macaulay.

The proofs utilize results of interest in their own right:

Proposition A. Let $L$ be a field, $y_{0}, \cdots, y_{m}$ indeterminates over $L, S=L\left[y_{0}, \cdots, y_{m}\right]$, and $Y=\operatorname{Proj}(S)=\boldsymbol{P}_{L}^{m}$. Let $K$ be a subfield of $L$, and let $R$ be a finitely generated graded K-algebra with $R_{0}=K$. Let $h: R \rightarrow S$ be a K-homomorphism which multiplies degrees by $d$. Let $P$ be the irrelevant maximal ideal of $R$, and let $X=\operatorname{Proj}(R)$. Let $U=Y-V(h(P) S)$. Let $\varphi=h^{*}$ be the induced $K$-morphism from the quasi-projective L-variety $U$ to the projective $K$-scheme $X$. Then $\varphi_{i}^{*}: H^{i}\left(X, \mathcal{O}_{X}\right) \rightarrow H^{i}\left(U, \mathcal{O}_{U}\right)$ is zero for $i \geqq 1$.

Proposition $\mathrm{A}^{\prime}$. Let $(R, P)$ be a local ring of prime characteristic $p>0$ and let $h$ be a homomorphism of $R$ into a regular Noetherian domain $S$. Suppose that for a certain $i$ the local cohomology module $H_{P}^{i}(R)$ has finite length. Then if $i \neq 0$ or $h(P) \neq 0$, the induced homomorphism $H_{P}^{i}(R) \rightarrow$ $H_{P S}^{i}(S)$ is zero.

1. Applications and corollaries. We note that the Main Theorem is stronger than the prior conjectures $[2, \S 0]$ or $[3$, p. 56], where $S$ was

AMS (MOS) subject classifications (1970). Primary 14M05, 20G05, 14B15, 13D05; Secondary $13 \mathrm{H} 05,13 \mathrm{H} 10$.

${ }^{1}$ Both authors were supported in part by NSF grant GP-37689.

Copyright @ American Mathematical Society 1974 
assumed to be a polynomial ring over $K$ and the action was assumed to preserve the grading. (This issue was first raised in [5] and [6].)

Second, we observe that the main result of [2] (where $G$ was $\operatorname{GL}(1, K)^{m}$ ), and, in characteristic 0 , the main results of [6], the roughly equivalent papers [3], [10], [11] (dealing with the Cohen-Macaulay property for Schubert varieties), and the thesis [9] all follow at once from the Main Theorem here.

Third, we note that the Main Theorem implies that Serre-Grothendieck duality will hold in a useful form (i.e. the pairing will be nonsingular: cf. [1, pp. 5 and 6]) for many orbit spaces of actions of linearly reductive groups on nonsingular varieties.

Fourth, we observe some ideal-theoretic corollaries. Let $S=$ $\left[y_{0}, \cdots, y_{m}\right]$, a polynomial ring, and let $G$ act so as to preserve degrees. Then $R=S^{G}$ will be generated over $K$ by finitely many forms of $S$, and we can write $R \cong K\left[z_{0}, \cdots, z_{t}\right] / I$. Here, we assume that $z_{0}, \cdots, z_{t}$ map to generating forms of $R$, and we grade $T=K\left[z_{0}, \cdots, z_{t}\right]$ so that the $K$-homomorphism preserves degrees. Then $I$ will be a homogeneous ideal of $T$, and is the solution to the "second main problem of invariant theory" (cf. [14, Chapter II, C]) for this particular representation. In this situation the assertion that $R(\cong T / I)$ is Cohen-Macaulay is equivalent to the assertion that $I$ is perfect, i.e. $\operatorname{pd}_{T} T / I=$ grade $I$. Let $\mathscr{K}$ be a graded $T$-free minimal resolution of $T / I$. Then from the Main Theorem and, for example, Theorem 3 and Corollary 1.2 of [4], we have

COROLlary 1. With notation as above, so that $S^{G} \cong T / I$, the length of $\mathscr{K}$ is $g=$ grade $I=$ height $I$, and $\mathscr{K}$ is grade-sensitive. That is, if $u_{0}, \cdots$, $u_{t}$ are elements of a Noetherian $K$-algebra $B$, and we make $B$ into a $T$ algebra by means of the homomorphism $h$ which takes $z_{i}$ to $u_{i}, 0 \leqq i \leqq t$, then if $J=h(I) B$ and $E$ is any $B$-module of finite type such that $J E \neq \bar{E}$, then the grade of $J$ on $E$ is the number of vanishing homology groups, counting from the left, of the complex $\mathscr{K} \otimes_{T} E$. In particular, if the grade of $J$ on $E$ is equal to $g$, then $\mathscr{K} \otimes_{T} E$ is acyclic.

Corollary 2. With notation as in Corollary 1 , let $E=B$. Then every minimal prime of $J=h(I) B$ has height at most $g$, and if the grade of $J$ is as large as possible, i.e. $g$, then $J$ is perfect $\left(\mathscr{K} \otimes_{T} B\right.$ is acyclic and gives a resolution of length $g$ ) and hence all the associated primes of $J$ have grade g. If $J$ has grade $g$ and $B$ is Cohen-Macaulay, then the associated primes of $J$ all have height $g$ and $B / J$ is again Cohen-Macaulay.

We also note

COROLlary 3. If $K$ has characteristic 0 and $G$ is semisimple and acts on $S=K\left[y_{0}, \cdots, y_{m}\right]$ so as to preserve the grading, then $R=S^{G}$ is a CohenMacaulay UFD and, hence, Gorenstein. 
3. Remarks on the proof of the Main Theorem. When $G$ is linearly reductive there is an $R$-module retraction (the Reynolds operator) of $S$ onto $R=S^{G}$. This fact is crucial in the proof of the Main Theorem. The proof goes roughly like this: First, we reduce to the case where $G$ is connected and then the theorem is stated slightly more generally- $S$ is only assumed regular at $G$-invariant primes. Utilizing devices involving associated graded rings and generalized Rees rings we make a reduction to a sort of "minimal" graded case: $S$ is the symmetric algebra of a projective module $E$ over a domain $B$ (where $G$ acts on $B, E$ and $B$ is a $K$ algebra) and $B$ has no $G$-invariant ideals except $0, B . R=S^{G}$ is a finitely generated graded algebra over a field, $B^{G}$, and $R_{\mathscr{P}}$ is Cohen-Macaulay except possibly when $\mathscr{P}=P$, the irrelevant maximal ideal (this last condition is what we meant by "minimal"). Let $L$ be the field of fractions of $B$. Then $L \otimes_{B} S=L\left[y_{0}, \cdots, y_{m}\right]$ is a polynomial ring. $R$ is a direct summand of $S$ (via the Reynolds operator) and this turns out to imply that $R$ is pure in $L \otimes_{B} S$. Because of this purity, the maps described in Proposition $\mathrm{A}$, which are zero by Proposition $\mathrm{A}$, are also injective, and one finds that $H^{i}\left(X, \mathcal{O}_{X}\right)=0, i \geqq 1$, where $X=\operatorname{Proj}(R)$. [We note that Proposition A itself is proved by a reduction to characteristic $p$.] By "minimality" the local rings of $X$ are Cohen-Macaulay and one can use SerreGrothendieck duality to show that $R^{(d)}=\sum \bigoplus_{n \geq 0} R_{n d}$ is Cohen-Macaulay for all large $d$. In the final stages of the proof, we show that $R$ itself is Cohen-Macaulay by reducing to characteristic $p$ a second time. (An important point is that in characteristic $p$ we can take $d=p^{e}$ for large $e$ and then embed $R \rightarrow R^{(d)}$ by using the Frobenius.) A key technical lemma which we use repeatedly in the reductions to characteristic $p$ and which generalizes the usual statements about generic flatness is

Lemma. Let $A$ be a Noetherian domain, $R$ an A-algebra of finite type, $S$ an $R$-algebra of finite type, $E$ an $S$-module of finite type, and $M$ an $R$-submodule of $E$ of finite type. Then there is an $a \in A-\{0\}$ such that $E_{a} / M_{a}$ is $A_{a}-$ free.

The proof of the characteristic $p$ Theorem is easier and uses local cohomology analogues of the arguments in the proof of the Main Theorem.

\section{Concluding remarks.}

REMARK 1. The regularity of $S$ is essential in the statement of the Main Theorem. There are counterexamples when $G=\mathrm{GL}(1, K)$ and $S$ is a graded Cohen-Macaulay Gorenstein UFD. But the regularity of $S$ is used in an apparently rather nongeometric way: it is only used to show that the Frobenius in a certain auxiliary ring, after reducing to characteristic $p>0$, is flat (cf. [8]). 
REMARK 2. The fact that reduction to characteristic $p$ seems to be essential in the proof of the Main Theorem is odd, because the Main Theorem is primarily a characteristic 0 theorem. There are very few linearly reductive groups in characteristic $p>0$. See [12]. We note that in [13] techniques related to ours are used to settle a number of other questions.

\section{REFERENCES}

1. A. Altman and S. Kleiman, Introduction to Grothendieck duality theory, Lecture Notes in Math., vol. 146, Springer-Verlag, Berlin and New York, 1970. MR 43 \#224.

2. M. Hochster, Rings of invariants of tori, Cohen-Macaulay rings generated by monomials, and polytopes, Ann. of Math. (2) 96 (1972), 318-337.

3. - Grassmannians and their Schubert subvarieties are arithmetically CohenMacaulay, J. Algebra 25 (1973), 40-57.

4. - Grade-sensitive modules and perfect modules, Proc. London Math. Soc. (to appear).

5. M. Hochster and J. A. Eagon, A class of perfect determinantal ideals, Bull. Amer. Math. Soc. 76 (1970), 1026-1029. MR 42 \#1814.

6. - Cohen-Macaulay rings, invariant theory, and the generic perfection of determinantal loci, Amer. J. Math. 93 (1971), 1020-1058.

7. M. Hochster and J. L. Roberts, Rings of invariants of reductive groups acting on regular rings are Cohen-Macaulay, Advances in Math. (to appear).

8. E. Kunz, Characterization of regular local rings for characteristic p, Amer. J. Math. 91 (1969), 772-784. MR 40 \#5609.

9. R. E. Kutz, Cohen-Macaulay rings and ideal theory in rings of invariants of algebraic groups, Thesis, University of Minnesota, Minneapolis, Minn., 1971.

10. D. Laksov, The arithmetic Cohen-Macaulay character of Schubert schemes, Acta Math. 129 (1972), 1-9.

11. C. Musili, Postulation formula for Schubert varieties, J. Indian Math. Soc. 36 (1972), 143-171.

12. M. Nagata, Complete reducibility of rational representations of a matric group, J. Math. Kyoto Univ. 1 (1961/62), 87-99. MR 26 \#236.

13. C. Peskine and L. Szpiro, Dimension projective finie et cohomologie locale, Thesis, Orsay, 1971 (Série A, No. d'ordre 781), Inst. Hautes Études Sci. Publ. Math. 42 (1973), 323-395.

14. H. Weyl, The classical groups. Their invariants and representations, 2nd ed., Princeton Univ. Press, Princeton, N.J., 1946.

Department of Mathematics, University of Minnesota, Minneapolis, Minnesota 55455

Current address (Melvin Hochster): Mathematisch Institut, Aarhus University, Aarhus, Denmark

(After July 1, 1974) Department of Mathematics, Purdue University, West LaFayette, Indiana 47907 